\title{
EVALUATION OF MOVING LEAST SQUARES AS A TECHNIQUE FOR NON- RIGID MEDICAL IMAGE REGISTRATION
}

By

Vijayalakshmi Sathyanarayanan

\author{
Thesis \\ Submitted to the Faculty of the \\ Graduate School of Vanderbilt University \\ in partial fulfillment of the requirements \\ for the degree of
}

\section{MASTER OF SCIENCE}

in

Electrical Engineering

December, 2008

Nashville, Tennessee

Approved:

Professor Robert E. Bodenheimer

Professor Benoit M. Dawant 


\section{ACKNOWLEDGEMENTS}

I would like to thank my advisor Dr. Robert E. Bodenheimer for giving me the opportunity to work with him and also for guiding me through the course of my thesis work. I would also like to thank Dr. Benoit Dawant for helping me shape my work and giving valuable suggestions which helped complete this thesis.

Last but not the least, I would like to express my thanks to my family especially my sister who has been with me in each step that I took, for their continuous encouragement which was essential for me to progress. 


\section{TABLE OF CONTENTS}

Page

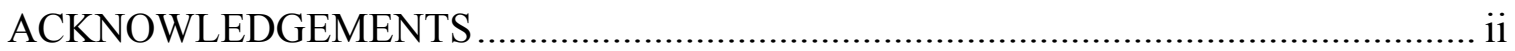



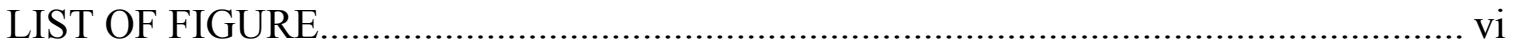

Chapter

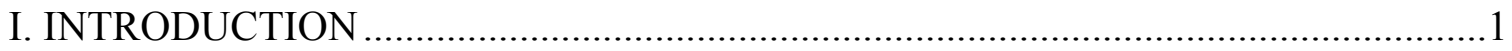

II. BACKGROUND

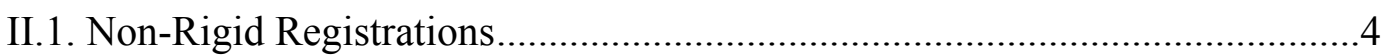

II.2. As-Rigid-As-Possible Transformations ……….........................................5

II.3. Thin-plate Spline Transformations...............................................................

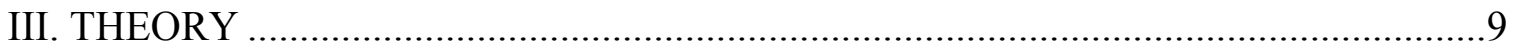

III.1. Moving Least Squares transformations .........................................................

III.1.1. 2D transformation using Moving Least Squares ...........................10

III.1.2. 3D transformation using Moving Least Squares ...........................12

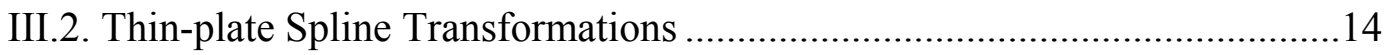

III.2.1. 2D Thin-plate Spline transformation ............................................15

III.2.2. 3D Thin-plate Spline transformation ............................................16

III.3. Computational aspects of MLS and TPS algorithms ...................................17

IV. EVALUATION OF TWO DIMENSIONAL REGISTRATIONS USING MLS

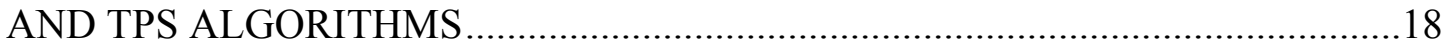

IV.1. 2D CT Images of whole body mice ……….............................................18

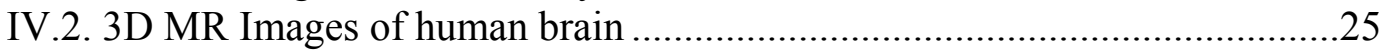

V. EVALUATION OF THREE DIMENSIONAL REGISTRATIONS USING MLS AND TPS ALGORITHMS........................................................ 31

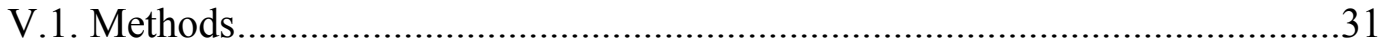

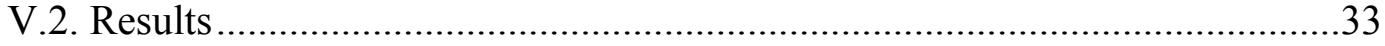


VI. CONCLUSION AND FUTURE WORK.

REFERENCES .. 


\section{LIST OF FIGURES}

Figure

1. Registration of 2D CT mice. (a) Source image with 35 control points. (b) Target image with 35 control points

2. Zoomed section of the source showing spacing in the spine. The MLS technique does not cause the odd stretching....

3. Registration of 2D CT mice. (a) Source image with 25 control points. (b) Target image with 35 control points

4. Registration of 2D CT mice. (a) Source image with 25 control points. (b) Target image with 10 control points.

5. Schematic representation of $2 \mathrm{D}$ registration of MR brain images.....

6. Registration of 2D MR images of the brain. (a) Source image (b) Target image with 19 control points. (c) Registration

7. Color coded TRE maps superimposed on brain registration results of (a) MLS and (b) TPS. The MLS image

8. Registration of 3D CT volumes of mice. (a),(b): 4 slices from source and target volumes (c) 4 slices from

9. (a) 4 slices from source volume. (b) 4 slices from target volume. (c) 4 slices from MLS registered result superimposed on 


\section{LIST OF TABLES}

Table

Page

1. Computational time for MLS and TPS algorithms in seconds....................17

2. Mean, standard deviation and T-test results of TRE for points A, B and C in

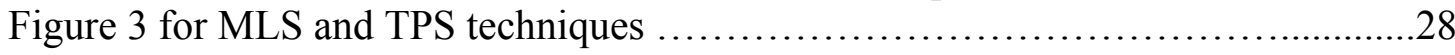

3. Mean and standard deviation of difference (TPS-MLS) in TRE for MLS and TPS

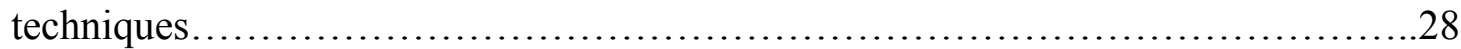




\section{CHAPTER I}

\section{INTRODUCTION}

Image registration is the process of aligning two images such that it is possible to determine corresponding points in them. Image registration is used extensively in the medical field for monitoring disease progression, image guided surgery, studying brain shift after surgery and developing medical atlases. The process for image registration involves two images-the source and the target images - and finding the best deformation field to align the two images. Various features like points, line segments and intensities can be used as the basis for the registration. A detailed classification can be found in Sonka and Fitzpatrick [2000]. Functions used to map the source image to the target image are called transformation functions. Image registration is classified as rigid or non-rigid based on the transformation function used. The application determines whether rigid or non-rigid registration is the most appropriate technique.

Rigid registrations are those in which the distances between all points remain constant before and after the registration. This type of image registration is popular since it involves a rotation and translation alone to achieve the mapping between two images. Rigid registrations have the advantage of being simple and easy in the sense that it is possible to predict how the transformation will perform. The most common application for rigid registrations is to register images obtained from same subject over a short duration of time. 
Non-rigid registrations are those in which distance between all points do not necessarily remain constant after the registration. Unlike rigid registrations, non-rigid registrations involve more complex computations like local stretching and scaling to map the two images. Applications requiring some local scaling and stretching cannot use rigid registrations and hence use non-rigid registrations. An example of such an application would be registering images obtained from two subjects where anatomical differences exist or to monitor disease progression [Freeborough and Fox 1998].

This thesis focuses on non-rigid registrations, which are still an area of ongoing research. The main drawback of non-rigid registration is the unpredictable nature of the deformation. It is not possible to exactly specify the mapping of each point in the source image to the target image. It is also possible that such registrations can cause certain regions, which should remain rigid for underlying physical reasons, to deform due to scaling or shearing. It would thus be good to have a non-rigid registration technique that produces local deformations only where needed while still preserving the overall rigidity of the transformation as much as possible. Such transformations are called as-rigid-aspossible. Fortunately such techniques exist, and this thesis focuses on a one method called the Moving Least Squares (MLS) transformation. This technique is relatively recent and is better know for surface reconstruction [Kolluri et al. 2005] and in computer graphics for image deformation and morphing [Schaefer et al. 2006] but has not been previously applied to medical images previously.

The goal then, of this thesis is to apply the MLS technique to two and three dimensional medical images where rigid transformations are not suitable and compare the results to similar non-rigid registration techniques. MLS is a point-based technique in 
which two images are aligned based on feature points extracted from them, and therefore we compare it to another popular point-based non-rigid method, Thin-plate Spline (TPS) transformation [Bookstein 1989; Goshtasby 1988]. Thus, this thesis assesses the applicability of the MLS technique as a general point based non-rigid registration technique and compares it to another widely used technique both qualitatively and quantitatively.

This thesis is organized as follows. Chapter II discusses the related work in nonrigid registrations and the MLS and TPS techniques. Chapter III describes the theory behind the Moving Least Squares and the Thin-plate Spline techniques in detail for 2and 3- dimensional data. Chapters IV and V describe the method and the results for registering 2- and 3- D data using the two techniques. We conclude with a comparison of the two methods based on the results and future possibilities of using the MLS method in Chapter VI. 


\section{CHAPTER II}

\section{BACKGROUND}

This chapter describes previous work in non-rigid image registration. We also describe related work in as-rigid-as-possible transformations as well as work in medical image registration using the thin-plate splines transformations.

\section{II.1 Non-rigid Image registration}

Medical image registration is a very popular area of image processing with applications ranging from monitoring disease progression [Freeborough and Fox 1998], building medical atlases [Toga and Thompson 2000], to image guided surgery [Sauer 2005] to name a few. Maintz and Viergever [1998] provide a survey of the recent publication in the field of medical image registration. A detailed theory on the various aspects of medical image registration including algorithms their validation and applications can be found in Fitzpatrick and Sonka [2000]. More details about the feature selection and correspondence, transformation functions and evaluation methods for 2-D and 3-D image registration can be found in Goshtasby [2005]. Non-rigid registration in an active area of research in the field of medical image registration. This activity is due to the fact that one cannot decide on the best algorithm for all applications. Each algorithm works well under certain constraints and conditions but may not do so under a different set of conditions. Crum [2004] discusses the various types of non-rigid registration 
algorithms, their concepts, applications and limitations. The paper also provides literature references in the area of non-rigid registrations.

The problem faced in non-rigid registrations is the fact that there exists no universal solution for the mapping problem [Hajnal et al. 2001]. Another drawback for these registrations is the inability to validate results due to the lack of a reference standard to compute the exact errors obtained in the registration process [Hajnal et al. 2001]. The main problem that non-rigid registrations pose is that it is not possible to actually predict the deformation field [Crum et al. 2004]. It is true that it is possible to map the control points exactly in the source and target images/volume using different methods [Schaefer et al. 2006; Goshtasby 1988; Bookstein 1999]. However it is not possible to predict where the other points in the source image/volume map onto in the target image/volume. This is because non-rigid registration involve more than a rotation and a translation as required for rigid registrations [Sonka and Fitzpatrick 2000; Crum et al. 2004]. This thesis focuses on evaluating a non-rigid registration technique which allows the deformation field to be as-rigid-as-possible while still performing local stretching deformations. The following section describes related work in as-rigid-aspossible shape transformations.

\section{II.2 As-rigid-as-possible Transformations}

The concept of as-rigid-as-possible transformations was first introduced by Alexa et al. [2000]. This paper presents an object space morphing technique that blends the interiors of objects as a smooth blending. This technique involves triangulating the object 
before applying the morphing algorithm. This morphing was called as-rigid-as-possible because the objects undergo minimum distortion during morphing. The shape manipulation technique presented by Igarashi et al. [2005] is also based on as-rigid-aspossible transformations. This technique also involves triangulating the source object and solving a linear system of equations with the number of equation equal to the number of vertices in the triangular mesh.

The non-rigid registration technique evaluated in this thesis, the Moving Least Squares transformation is described in [Schaefer et al. 2006]. This paper builds on the technique described by Igarashi but aims at achieving faster deformations. This paper proposes the transformation of objects by using linear moving least squares. No triangulation of the input is needed in this case and the transformation can be computed at each point in the image. The transformations achieved using rigidity constraints are asrigid-as-possible with minimum non-linear shearing and non-uniform scaling. Also, the system is simple to solve in case of $2 \mathrm{D}$ images as it results in a simple linear $2 \times 2$ system. The MLS technique can also be applied to 3D volumes and this extension though causes the system to lose its quadratic nature seen in the $2 \mathrm{D}$ case does not involve a lot of complex computations. Chapter III contains the detailed theory behind the MLS transformation for both $2 \mathrm{D}$ and $3 \mathrm{D}$ applications. The resulting transformations in both applications are smooth and involve the least amount of shearing and non-uniform scaling. This property of the MLS algorithm make it a suitable candidate for applications in medical image registration where rigid registrations are insufficient and non-rigid registrations are necessary to align features of the image. Using the MLS technique 
ensures that local deformations are achieved while retaining the overall rigidity of the object.

\section{II.3 Thin-Plate Spline Transformations}

Thin-plate splines are a part of the spline family with radial basis functions as the interpolating function. They produce smooth and closed form transformations and have been used extensively in image deformation. The detailed theory behind the TPS transformation for 2D and 3D applications can be found in Chapter III. The remaining part of this section describes some of the work done in medical image registration using the TPS transformation.

Thin-plate Splines have been used in remote sensing for mapping images. Goshtasby [1988] describes how the thin-plate splines can be used in image deformation for remote sensing images. His book on $2 \mathrm{D}$ and $3 \mathrm{D}$ registrations also describes the TPS transformation function and it applications [Goshtasby 2005]. Though the TPS produces smooth transformations, the main disadvantage of the technique is that each control point in the image has a global effect on the transformation and thus even if one point is perturbed all other points in the image do not get mapped correctly [Crum et al 2004]. The work by Bookstein [1999] also describes the theory behind TPS transformations and how it can be used in medical image processing. Numerous papers have been published in the medical image processing area using the TPS algorithm in image registration. The TPS algorithm can be used to obtain an elastic transformation to map the source image to the target image. This technique has been described by Rohr et al. [2003]. Building 
medical atlases is one important application of image registration. The TPS algorithm can be used in constructing medical atlases and is described in detail by Park et al. [2003]. The TPS technique has also been used with intensity as the control feature to obtain consistent registrations and is described in the work by Johnson and Christensen [2001]. Rohr [2001] uses the TPS as a technique to obtain elastic registration of brain images taking into account the errors at the landmark points in the images. The method proposed in this paper is applicable to 2D and 3D MR images.

This thesis establishes that the MLS transformation technique can be used as an alternate to the TPS transformation technique and can perform better due to its as-rigidas-possible nature. This could enable the MLS technique to be used in applications like constructing medical atlases, studying the progression of diseases, post-operative shift of brain and other such applications where the TPS technique has been used so far. 


\section{CHAPTER IIII}

\section{THEORY}

This chapter discusses the theory behind the moving least squares and thin-plate spline techniques.

\section{III.1 Moving Least Squares Transformation}

The moving least squares technique was used in computer graphics initially for surface reconstruction [Kolluri et. al 2005]. It can also be used for the deformation of 2D and 3D objects [Schaefer et. al 2006; Cuno et. al 2007; Zhu and Gortler 2007]. The deformation is carried out by selecting control points on the source image. The points to which these control points must be mapped to in the target image are chosen as the deformed points. This presents the deformation as a registration problem. A transformation function must be computed to map each point in the source image to the corresponding point in the target image. The principle of the MLS technique is to minimize the least squares error function obtained during this transformation process. A transformation function is obtained for each point in the image and is based on a weight function included in the least squares error function at each point of evaluation. This weight function ensures that the effect of a control point is seen most in the regions immediately surrounding it, while its effect is less prominent in far off regions. The transformation matrix of the MLS technique can include affine, similarity and as-rigid-aspossible transformations. Affine transformations are those which preserve the parallel 
nature of lines in the image and also produce non-uniform scaling and skewing, Similarity transformations are a part of the affine transformations but with uniform scaling. As-rigid-as-possible transformations are those which are capable of producing local deformations while maintaining a global rigidity of the image.

This thesis focuses on the as-rigid-as-possible transformations. The transformation function is smooth and interpolates the control points. The following two sections describe the 2D and 3D transformations using MLS.

\section{III.1.1 2D Transformations using Moving Least Squares}

Given a set of control points on the source and target images, the MLS technique computes the transformation $l_{v}(x)$ that best minimizes the least squares error:

$$
\sum_{i}\left|l_{v}\left(p_{i}\right)-q_{i}\right|^{2}
$$

where $p_{i}$ and $q_{i}$ are the set of control points in the source and target images respectively. This transformation however produces a single affine transformation of the entire image as there is no control over the scaling or shearing in the image. A weighting function included to this least squares error fixes this problem and thus produces a different transformation function for each point of evaluation of the image.

$$
\sum_{i} w_{i}\left|l_{v}(p)-q_{i}\right|^{2}
$$

The weighting function $w_{i}$ is of the form

$$
w_{i}=\frac{1}{\left|p_{i}-v\right|^{2 \alpha}}
$$


where $v$ is the point of evaluation in the image and $\alpha$ is a parameter of the weighting function whose value decides if the weights computed are small or large. The weighting function $w_{i}$ is dependent on the point of evaluation and thus produces a different transformation for each point of the image. Hence the method is called Moving Least Squares. We can see that as $v$ approaches $p_{i}$, the weight approaches infinity and the transformation function interpolates.

The transformation function can be solved as a simple linear transformation matrix, $M$ and a translation vector, $T$ as

$$
l_{v}(x)=x M+T
$$

The transformation matrix $M$ can be modified to include affine, similarity and rigid transformations. To perform as-rigid-as-possible transformations, the matrix, $M$ must be constrained to satisfy the condition for rigidity $M^{\prime} M=I$. The translation component can be easily computed by

$$
T=q_{*}-p_{*} M
$$

where $p_{*}$ and $q_{*}$ are the weighted centroids of the control points given by

$$
p_{*}=\frac{\sum_{i} w_{i} p_{i}}{\sum_{i} w_{i}} \text { and } q_{*}=\frac{\sum_{i} w_{i} q_{i}}{\sum_{i} w_{i}}
$$

The transformation function can now be calculated as

$$
l_{v}(x)=\left(x-p_{*}\right) M+q_{*}
$$

The least squares problem can be written as $\sum_{i} w_{i}\left|\hat{p}_{i} M-\hat{q}_{i}\right|^{2}$

where $\hat{p}_{i}=p_{i}-p_{*}$ and $\hat{q}_{i}=q_{i}-q_{*}$ 
The transformation matrix for the as-rigid-as-possible transformations can be obtained by eliminating the scaling constant. The solution is simple and closed form. It can be obtained easily by a slight modification of the similarity transformation for which the transformation matrix must satisfy the condition $M_{1}^{\prime} M_{2}=M_{1} M_{2}^{\prime}=\lambda^{2} I$, where $\lambda$ is some constant and $M_{1}$ and $M_{2}$ are the columns of $\mathrm{M}$ and are vectors of size $2 \times 1 . M_{1}$ and $M_{2}$ have the relationship $M_{2}=M_{1}^{\perp}$ such that $(x, y)^{\perp}=(-y, x)$. For rigidity condition to be satisfied $M^{\prime} M=I$, the scaling constant needs to be removed. By using partial derivatives with respect to the free variables in $M$ and substituting the values back into the error function the optimum transformation function is obtained as

$$
M=\frac{1}{\mu_{r}} \sum_{i} w_{i}\left(\begin{array}{c}
\hat{p}_{i} \\
-\hat{p}_{i}^{\perp}
\end{array}\right)\left(\begin{array}{ll}
\hat{q}_{i}^{T} & -\hat{q}_{i}^{\perp T}
\end{array}\right)
$$

where $\mu_{r}=\sqrt{\left(\sum_{i} w_{i} \hat{q}_{i} \hat{p}_{i}^{T}\right)^{2}+\left(\sum_{i} w_{i} \hat{q}_{i} \hat{p}_{i}^{\perp T}\right)^{2}}$ removes any scaling and thus produces an asrigid-as-possible transformation. The detailed derivation for the as-rigid-as-possible transformations can be seen in Schaefer et. al 2006.

\section{III.1.2 3D Transformations using MLS}

This section discusses the extension of the MLS technique to $3 \mathrm{D}$ volumes. The derivation for the transformation matrix involves more complex computations than the $2 \mathrm{D}$ derivations. The inclusion of the third dimension causes the system to no longer be quadratic in nature though it is still possible to obtain closed form solutions. The solution involves finding the best transformation minimizing the weighted least squares problem 
$\sum_{i} w_{i}\left|l_{v}(p)-q_{i}\right|^{2}$ and finding the optimal axis of rotation and the angular parameters. The procedure for selecting points is the same as in the $2 \mathrm{D}$ case but the points need to be selected in a volume thus including a third coordinate. Control point sets $\mathrm{P}$ and $\mathrm{Q}$ are selected from the source and target volumes. In order to obtain as-rigid-as-possible transformations, it is necessary that the transformation $l_{v}(x)$ is of the form

$$
l_{v}(x)=M x+T
$$

where $M$ is the transformation matrix and $T$ is the translation vector. The translation vector can be computed using partial derivatives as seen in section III.1 in equation (3.5) as $T=q_{*}-p_{*} M$, where $p_{*}$ and $q_{*}$ are the weighted centroids given by equation (3.6).

The least squares error problem can now be written as $\sum_{i} w_{i}\left|\hat{p}_{i} M-\hat{q}_{i}\right|^{2}$ as seen before in equation (3.8). Expanding this summation gives the following of which the second and third terms are constants.

$$
-2 \sum_{i} w_{i} \hat{q}_{i} M p_{i}^{T}+\sum_{i} w_{i}\left\|\hat{q}_{i}\right\|^{2}+\sum_{i} w_{i}\left\|\hat{p}_{i}\right\|^{2}
$$

Thus it can be seen that the minimization is achieved when $M$ can maximize the $\operatorname{term} \sum_{i} w_{i} \hat{q}_{i} M p_{i}^{T}$

The transformation matrix $M$ can be defined as a rotation of an angle around an axis. Let $\theta$ be the angle of rotation and $e$ be the axis of rotation. Applying such a rotation to a vector $v$ gives

$$
M_{e, \theta}\left(v^{T}\right)=e^{T} e v^{T}+\cos \theta\left(I-e^{T} e\right) v^{T}+\sin \theta\left|\begin{array}{ccc}
0 & -e_{z} & e_{y} \\
e_{z} & 0 & -e_{x} \\
-e_{y} & e_{x} & 0
\end{array}\right| v^{T}
$$


Now the problem of maximizing equation 3.14 becomes finding the best axis of rotation $e$ and angle of rotation $\theta$ maximizing

$$
\cos \theta \operatorname{Trace}(M)+(1-\cos \theta) e M e^{T}+\sin \theta V e^{T}
$$

where $R=\sum_{i} w_{i} q_{i}^{T} \hat{p}_{i}$ and $V=\sum_{i} w_{i} \hat{q}_{i} \times \hat{p}_{i}$

The solution for the axis of rotation and angle of rotation can be computed by applying Kuhn-Tucker optimality conditions to obtain an eigenvalue problem. Solving this eigenvalue problem provides the axis of rotation $e$ as the eigenvector of the matrix $M+M^{T}+c V^{T} V$ where $\mathrm{c}$ is the root of a fourth degree polynomial [Cuno et. al 2007] having closed form solution. The angle of rotation can be computed once the axis is found as

$$
\cos \theta=\frac{1-\|u\|^{2}}{1+\|u\|^{2}} \text { and } \sin \theta=\frac{-2\|u\|}{1+\|u\|^{2}}
$$

where $e$ is the unit vector of $u$.

The detailed derivation for the rotation axis and angle of rotation can be found in Cuno et al. [2007].

\section{III.2 Thin-Plate Spline Transformations}

The thin-plate splines are a part of the spline family with radial basis functions as the interpolating function. The equation for TPS contains an affine part as well as a nonaffine part with the interpolating function. This section discusses the equations required to deform 2D and 3D objects using TPS. 


\section{III.2.1 2D Thin-plate Spline Transformations}

The 2D thin-plate spline interpolating a set of control points is given by

$$
\begin{aligned}
& x^{\prime}=a_{0}+a_{1} x+a_{2} y+\sum_{i=1}^{N} F_{i} r_{i}^{2} \ln r_{i}^{2} \\
& y^{\prime}=b_{0}+b_{1} x+b_{2} y+\sum_{i=1}^{N} G_{i} r_{i}^{2} \ln r_{i}^{2}
\end{aligned}
$$

where $r_{i}^{2}=\left(x-x_{i}\right)^{2}+\left(y-y_{i}\right)^{2}, N$ represents the number of control points and $x_{i}$ and $y_{i}$ represent the coordinates of the $\mathrm{i}^{\text {th }}$ control point and I ranges from 1 to $N$.

The two equations specified above have $N+3$ unknowns each. The solution to obtain the deformation field is simple. $N$ equations can be formed by substituting the coordinates of the control points into the equations (3.17) and (3.18). Apart from these there are three constraints used for solving the transformation.

$$
\begin{aligned}
& \sum_{i=1}^{N} F_{i}=0 \\
& \sum_{i=1}^{N} x_{i} F_{i}=0 \\
& \sum_{i=1}^{N} y_{i} F_{i}=0
\end{aligned}
$$

Thus there are now $N+3$ equations to find out the $N+3$ unknowns. The last three constraints ensure that the surface being deformed does not rotate under the influence of the control points. With the help of these equations it is possible to solve for the transformation which is then applied to deform the source image. It should be noted here that the transformation maps the control points in the source image exactly onto the control points in the target image. However the other points in the image do not map exactly producing errors that become more pronounced in regions away from the control 
points. TPS have been observed to produce more errors while mapping images having local geometric differences. The symmetric nature of the logarithmic function causes the errors to be minimal in cases where the fiducial points are distributed symmetrically while asymmetrically distributed points produce more errors in the transformation.

\section{III.2.2 3D Thin-Plate Splines}

TPS can be extended to three dimensional volumes as well. In this case the transformation requires the computation of three components: $\mathrm{x}, \mathrm{y}$ and $\mathrm{z}$ to accommodate the three dimensions.

The transformation in this case is denoted as follows:

$$
\begin{gathered}
x^{\prime}=a_{0}+a_{1} x+a_{2} y+a_{3} z+\sum_{i=1}^{N} F_{i} r_{i} \\
y^{\prime}=b_{0}+b_{1} x+b_{2} y+b_{3} z+\sum_{i=1}^{N} G_{i} r_{i} \\
z^{\prime}=c_{0}+c_{1} x+c_{2} y+c_{3} z+\sum_{i=1}^{N} F_{i} r_{i}
\end{gathered}
$$

Where $r_{i}^{2}=\left(x-x_{i}\right)^{2}+\left(y-y_{i}\right)^{2}+\left(z-z_{i}\right)^{2}$

This equation has $N+4$ unknowns. As in the case of $2 \mathrm{D}$ splines $N$ equations can be obtained by substituting the control points in the above equation while 4 more equations can be obtained from the constraints which ensure the image does not rotate under the influence of the loads. The constraints are as follows: 


$$
\begin{aligned}
& \sum_{i=1}^{N} F_{i}=0 \\
& \sum_{i=1}^{N} x_{i} F_{i}=0 \\
& \sum_{i=1}^{N} y_{i} F_{i}=0 \\
& \sum_{i=1}^{N} z_{i} F_{i}=0
\end{aligned}
$$

Thus with $N+4$ equations for $N+4$ unknowns it is possible to solve for the deformation field to be applied to the image.

\section{III.3 Computational Aspects for MLS and TPS algorithms:}

We implemented both MLS and TPS in MATLAB. The MLS algorithm involves finding the transformation at each point in the image or volume and thus has a longer computation time than for TPS as seen in table 1. One way to reduce the computation time would be to decimate the image or volume using a grid and apply the deformation to each vertex of the grid instead of each point and interpolating the other points using bilinear interpolation. TPS transformations are computationally less time consuming.

Table 1. Computational time for MLS and TPS algorithms in seconds for 128x128 image.

\begin{tabular}{|c|c|c|}
\hline \multirow{2}{*}{ No. of Points } & \multicolumn{2}{|c|}{ Time for transformation in seconds } \\
\cline { 2 - 3 } & MLS & TPS \\
\hline 11 & 124 & 9 \\
\hline 26 & 236 & 15 \\
\hline 43 & 387 & 24 \\
\hline
\end{tabular}




\section{CHAPTER IV}

\section{TWO DIMENSIONAL REGISTRATION USING MLS AND TPS TRANSFORMATIONS}

This chapter presents the method and results for evaluation of two dimensional images using the MLS and TPS techniques. We use two dimensional Computed Tomography (CT) images and two dimensional Magnetic Resonance images for performing the registration.

\section{IV.1 2D CT Images}

We first used whole body CT images of mice to perform registrations using the two techniques. CT images show the bone structures more prominently than the soft tissue in the body. Since bones are rigid parts of the skeleton, CT images provide an excellent data set to evaluate the two techniques. The following sections discuss the datasets, the method for selecting the control points and the results obtained for the MLS and TPS techniques.

\section{IV.1.1 Method}

The CT images used for registration were obtained from two whole body CT volumes of two different mice. The volumes were of size $512 \times 512 \times 512$ with a voxel resolution of $0.2 \times 0.2 \times 0.2 \mathrm{~mm}^{3}$. A slice showing maximum detail was selected from each of the two volumes. These two slices were then used in the registration process. The head, 
spine and the legs were seen prominently in these two slices. This can be seen in Figure 4.1(a) and (b).

Selecting the control points is an important part of the registration process. We selected control points manually on the source image. These points were picked on the head, spine and legs due to their prominence in the CT image. Similarly points corresponding to these points or homologous points, which have same relative position on the target image with respect to the control point on the source image, were selected manually. This set of control points on the source and target images was used in the registration process using the MLS and TPS techniques. Figure 4.1 (a) and (b) shows one such set of homologous points on the source and target images.

\section{IV.1.2 Results}

We evaluated the performance of the two registration techniques based on the number of points used for registration and the placement of the points. Figure 4.1 (a) and (b) show the source and target images with homologous control points placed on the bony structures of the head, spine and legs. Figure 4.1 (c) and (d) show the result of the registration using MLS and TPS techniques. Figure 4.2 shows a zoomed in section of the spine of the source image of the mouse to show the already existing spacing in the spine. From the results we observed that the MLS technique produces a qualitatively better result than the TPS technique. The MLS registered result does not show the unnatural stretching seen in the spine. We also observed that the resulting transformation for the MLS technique maintains the overall rigidity of the image. This was attributed to the as- 

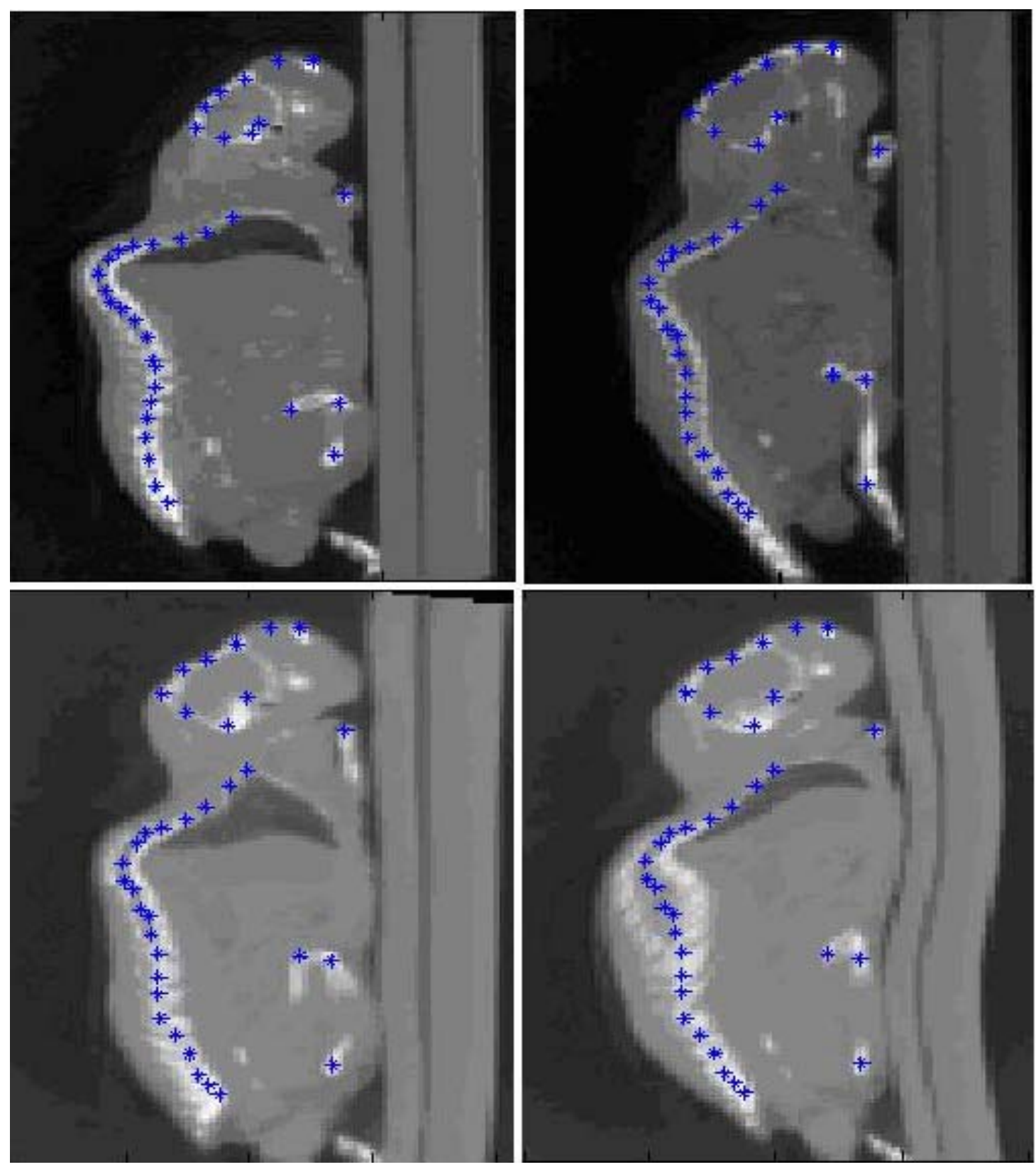

Figure 4.1: Registration of 2D CT mice. (a) Source image with 35 control points. (b) Target image with 35 control points. (c) Registration using MLS method.

(d) Registration using TPS method. The MLS method performs qualitatively better than the TPS method.

rigid-as-possible nature of the MLS algorithm. The ability to maintain the rigidity constraint by limiting the non-uniform scaling produces a deformation field which retains the overall rigidity of the image. 


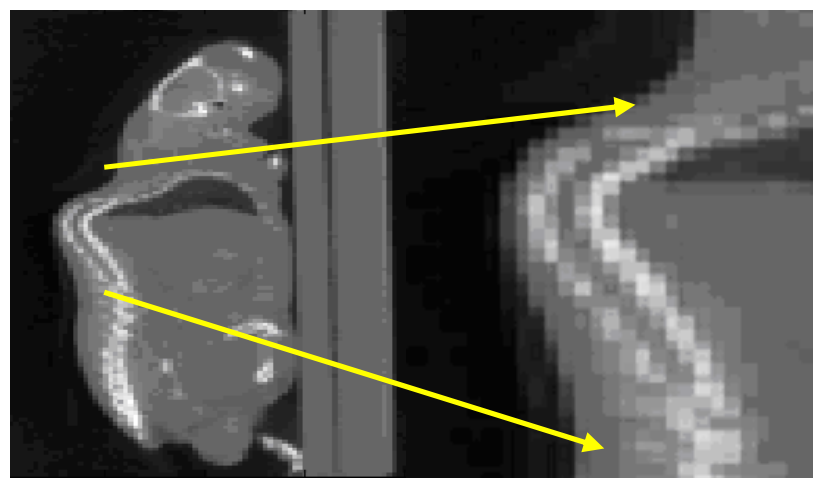

Figure 4.2. Zoomed section of the source showing spacing in the spine. The MLS technique does not cause the odd stretching of spine seen in the TPS technique as seen in Figure 1(d).
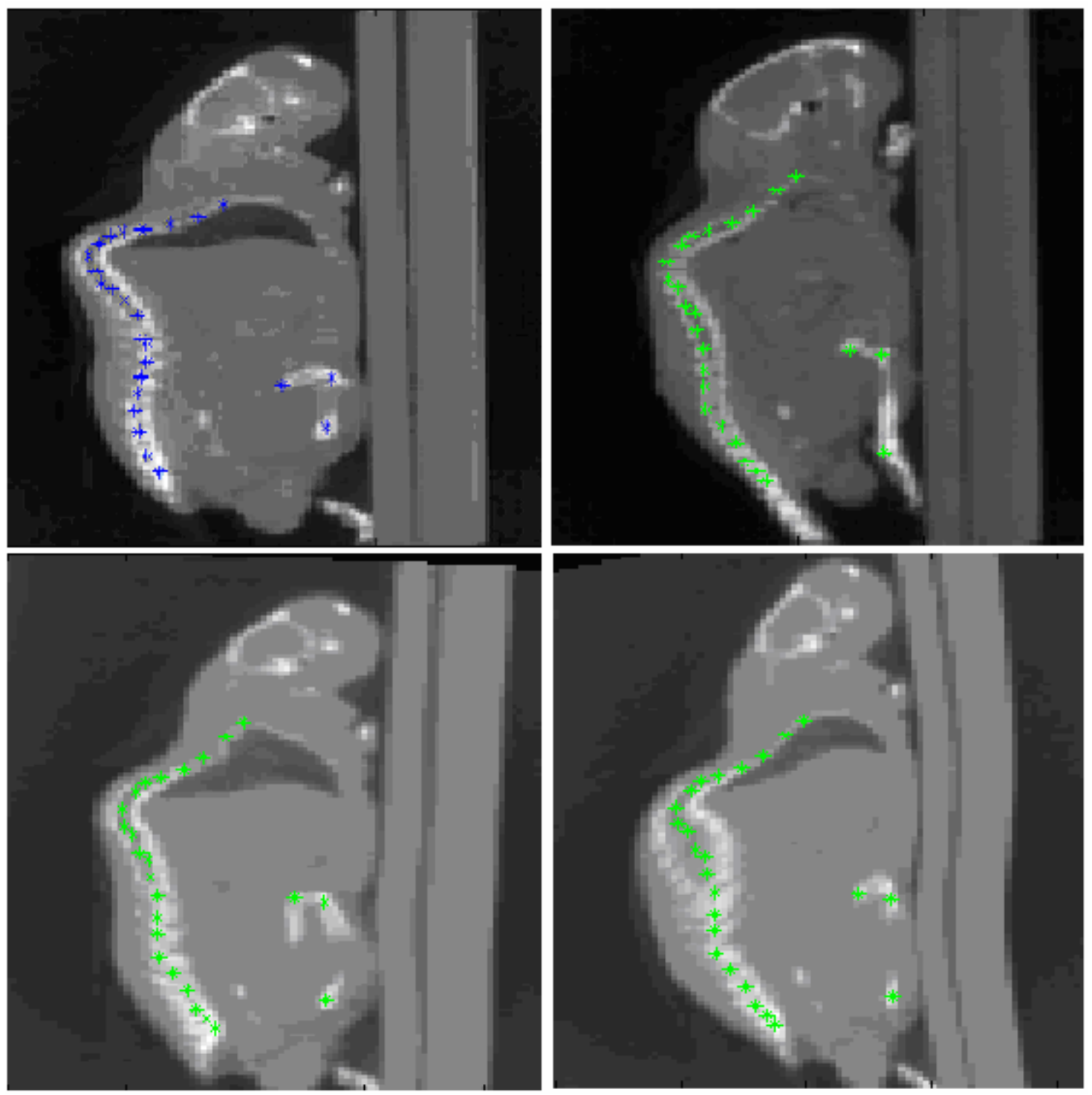

Figure 4.3: Registration of 2D CT mice. (a) Source image with 25 control points. (b) Target image with 35 control points. (c) Registration using MLS method.

(d) Registration using TPS method. The MLS method performs qualitatively better than the TPS method. 

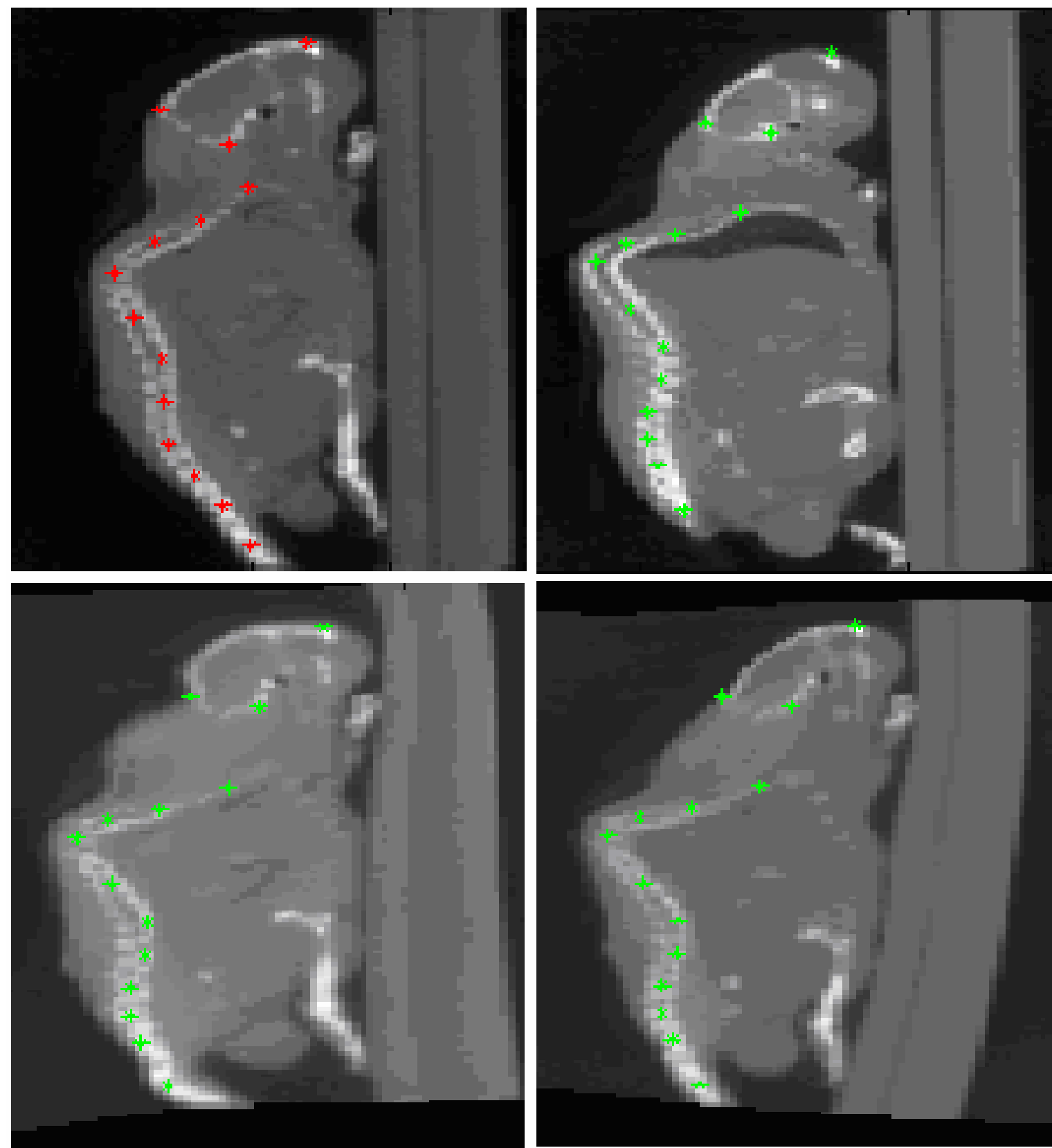

Figure 4.4: Registration of 2D CT mice. (a) Source image with 10 control points. (b) Target image with 10 control points. (c) Registration using MLS method.

(d) Registration using TPS method. The MLS method shows least stretching and scaling compared to the TPS method.

To evaluate the performance of the two techniques based on the placement of control points, we selected the control points only on the spine leaving out the head and the leg. Figure 4.3 (a) and (b) show the pair of source and target images with homologous 
control points placed only on the spine. The registration result using MLS and TPS techniques can be seen in Figure 4.3 (c) and (d). It was observed that even though control points were not placed in the head region, the MLS technique produced a qualitatively better result. This technique was not seen to cause any non-uniform scaling or stretching and also produces an as-rigid-as-possible transformation. The TPS transformation was seen to stretch the image unnaturally. In this case too, we observed the stretching caused in the spine region along with the stretching seen near the head region. Thus it can be said that non-uniform placement of control points affects the deformation field minimally in case of the MLS technique than the TPS technique which showed more stretching.

To show that MLS can be used as a better technique for non-rigid registration while maintaining the overall rigidity of the image we evaluated the two techniques by using a limited number of control points. Using more control points will produce equally good results for the two methods since this allows more control over the deformation field. Figure 4.4 (a) and (b) show the source and target images with 10 control points placed on the head and the spine. The results of the two registration techniques can be seen in the Figure 4.4 (c) and (d). A visual inspection of the two transformations showed that the MLS algorithm produces a qualitatively better image than the TPS method. The MLS transformation was observed to map the source to the target image with minimal stretching, due to its as-rigid-as-possible transformation ability, while the TPS transformation was observed to cause more stretching across the body of the mice. 


\section{IV.2. 2D MR human brain}

Registering MR brain images from two different patients requires non-rigid registration due to the presence of anatomical difference in the brain structures. We evaluated the performance of the two techniques by registering two corresponding depth two dimensional Magnetic Resonance (MR) image slices of the human brain. MR images provide more detail and greater contrast between the soft tissues in the body than CT images. The two slices used were 256x256 images of the sagittal view of the brain of two subjects. Such image from two subjects presents a lot of anatomical variations and thus provides a good dataset for non-rigid registration.

\section{IV.2.1 Method}

In case of brain images it is difficult to identify homologous control points in two images from different subjects. As these points play the most important role in the registration process it becomes necessary to accurately obtain homologous control points from the source and target images. We started with two slices S and T' from two different patients. The two slices were registered non-rigidly using the Adaptive Bases Algorithm (ABA) [Rohde et al. 2003] an intensity based method for registering two images or volumes. The deformation function thus obtained was applied to the source image $\mathrm{S}$ to get the new transformed image $\mathrm{T}$. $\mathrm{T}$ has homologous points to the source $\mathrm{S}$. This new image $\mathrm{T}$ was used as the target image in our registration process. $\mathrm{S}$ and $\mathrm{T}$ were registered using the MLS and TPS techniques. Figure 4.5 shows the diagrammatic representation of the process we followed to obtain the source and target images. 


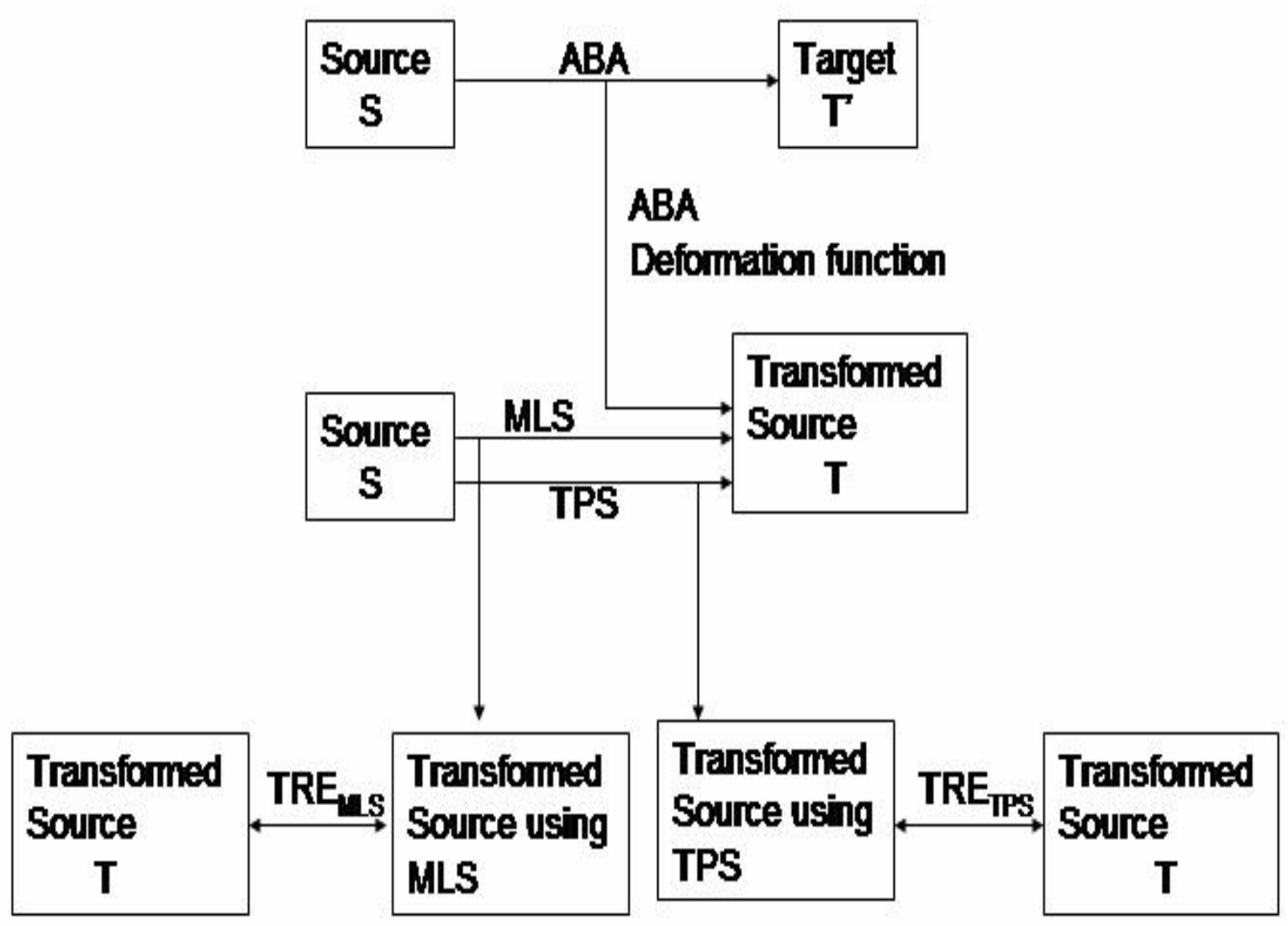

Figure 4.5: Schematic representation of 2D registration of MR brain images

\section{IV.2.2 Results}

We evaluated the performance of the two techniques qualitatively and quantitatively. Figure 4.6 (a) and (b) show the source and target images with homologous control points. We placed the control points on the skull, the tip of the nose, mouth and few control points were placed on the internal structures of the brain. The control points on the source image are shown in light blue color while the control points on the target image are shown in dark blue color. The results of the MLS and TPS techniques can be seen in Figure 4.6 (c) and (d). Visually both the methods look similar due to the intricate 
anatomy of the brain and it was noted that both methods perform acceptable transformations.

Since qualitative comparisons were not sufficient to evaluate the performance we performed quantitative evaluations as well. We computed the target registration error (TRE) at certain points in the image for both the methods. The target registration error at any point, which is nothing but the registration error at that point, is computed as the disparity in the position of two corresponding points after registration [Sonka and Fitzpatrick 2000; Hajnal et al. 2001]. A lower TRE thus indicates a better method. We performed 25 registration trials with 25 sets of control points. This was carried out to observe the error obtained in different registrations. To compute the TRE, we selected three points $\mathrm{A}, \mathrm{B}$ and $\mathrm{C}$ at random on the internal structures of the brain. We then computed the TRE at these points A, B and C, shown on the source image, by finding the points they mapped to on the MLS and TPS registered images. Since the points corresponding to $\mathrm{A}, \mathrm{B}$ and $\mathrm{C}$ could be found on the target image by using $\mathrm{ABA}$ we were able to compare the resulting TREs for the two methods. Table IV.1 shows the mean TREs for each of the points A, B and C. It was observed that the average TRE was lesser for the MLS method compared to the TPS method. The standard deviation of the error can also be seen in Table IV.1 and shows a lower value for the MLS method. We also performed a t-test at $95 \%$ confidence level to check if the means were statistically different. It was found that point A did not have a significant difference but points B and C had statistically different means. 

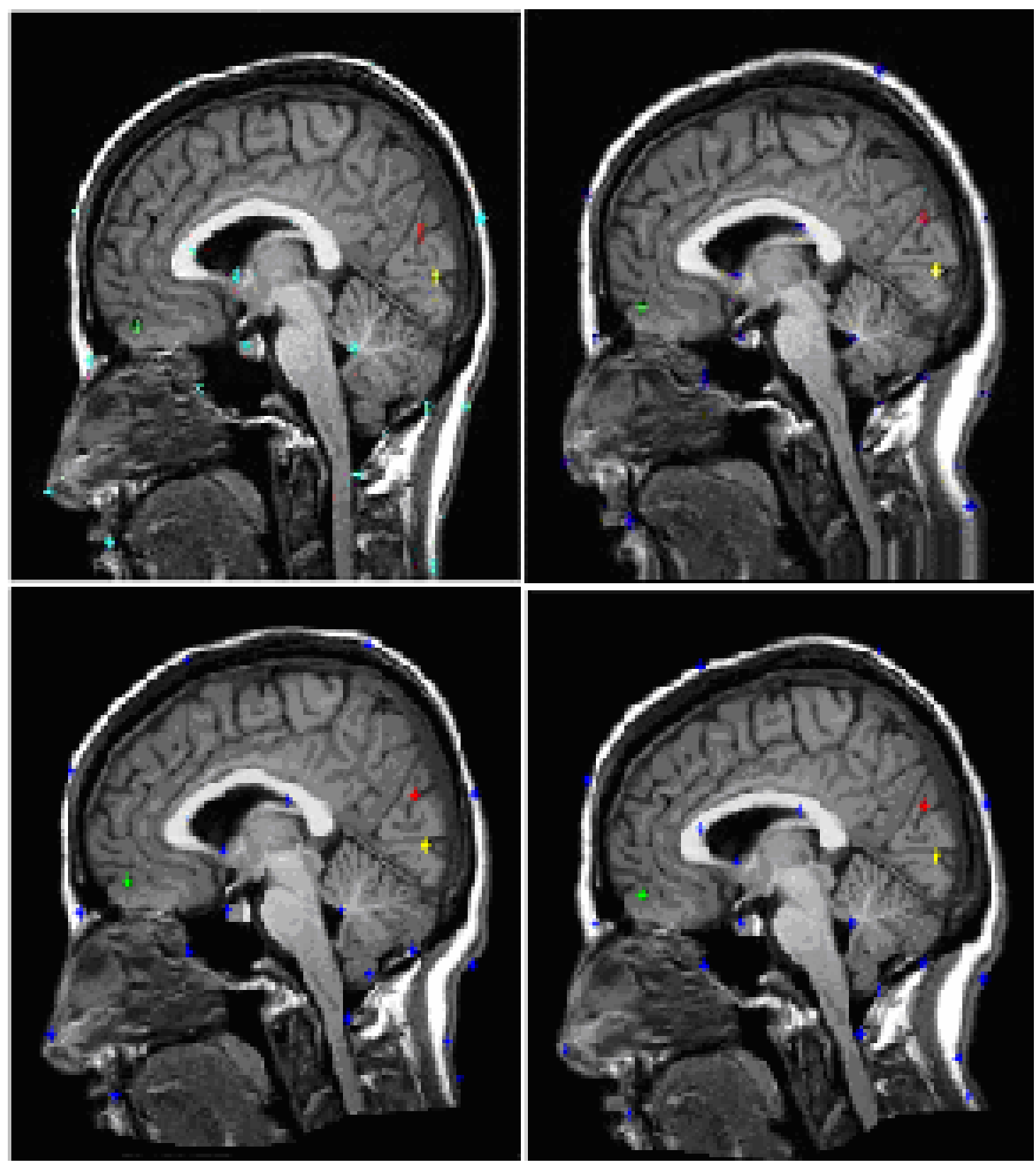

Figure 4.6. Registration of 2D MR images of the brain. (a) Source image (b) Target image with 19 control points. (c) Registration using MLS method. (d) Registration using TPS method. Points A, B and C are used in computing the target registration error for the two methods. 
Table IV.1. Mean, standard deviation and T-test results of TRE for points A, B and C in Figure 3 for MLS and TPS techniques over 25 registration trials. The t-test analysis shows that the mean is significantly different for points $\mathrm{B}$ and $\mathrm{C}$.

\begin{tabular}{|l|l|l|l|l|}
\hline \multirow{2}{*}{ MLS } & Point & $\mathrm{A}\left(\right.$ green*$\left.^{*}\right)$ & $\mathrm{B}\left(\right.$ red*$\left.^{*}\right)$ & $\mathrm{C}($ yellow*) \\
\cline { 2 - 5 } & Mean & 1.76 & 3.409 & 2.7133 \\
\cline { 2 - 5 } & Std. Dev & 1.3533 & 1.5369 & 1.4065 \\
\hline \multirow{2}{*}{ TPS } & Mean & 1.969 & 5.5942 & 4.1539 \\
\cline { 2 - 5 } & Std. Dev & 1.6567 & 2.3689 & 0.7056 \\
\hline \multicolumn{2}{|l|}{ Different? (t-test, 5\%) } & $\mathrm{N}$ & $\mathrm{Y}$ & $\mathrm{Y}$ \\
\hline
\end{tabular}

We also computed the mean and standard deviation of the difference in the TRE for the MLS and TPS methods i.e., (TRE $\left.\mathrm{TPS}_{\mathrm{T}}-\mathrm{TRE}_{\mathrm{MLS}}\right)$ as seen in Table IV.2. This was done to observe if the overall TRE was lower for the MLS method. To give and idea of the spatial variation of the TREs over these trials, we computed the mean TRE for the entire image and these values are presented as a color-scaled map onto the brain image as

Table IV.2. Mean and standard deviation of difference (TPS-MLS) in TRE for MLS and TPS techniques.

\begin{tabular}{|l|l|l|l|}
\hline Point & A(green*) & B(red*) & C(yellow*) \\
\hline Mean & 0.209 & 2.1851 & 1.4406 \\
\hline Std. Dev & 0.9748 & 1.8434 & 1.6039 \\
& & & \\
\hline
\end{tabular}



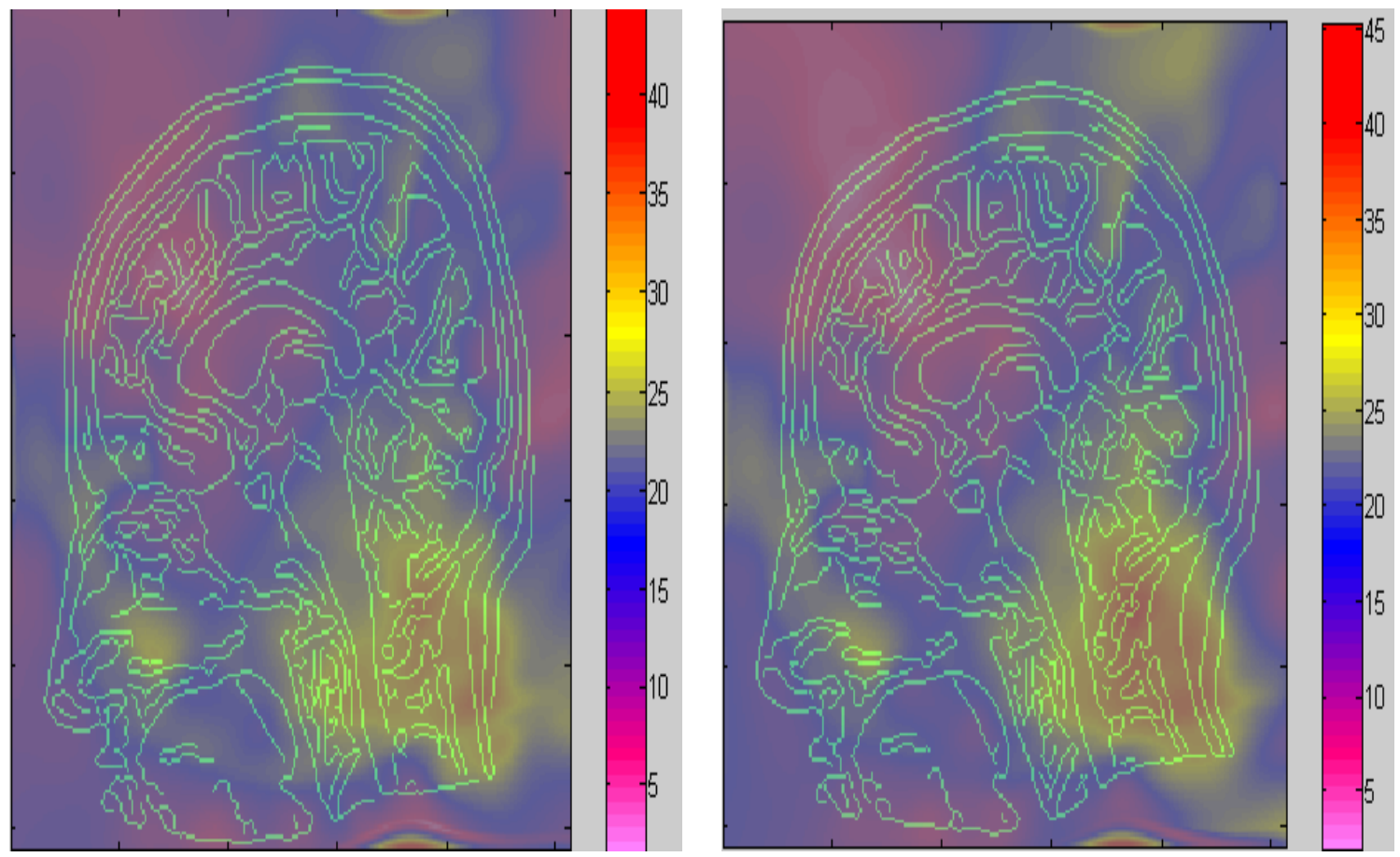

Figure 4.7. Color coded TRE maps superimposed on brain registration results of (a) MLS and (b) TPS. The MLS image has lower maximum and average TRE over the entire image.

shown in Figure 4.7. The color-scaled map shows that the TPS method has a larger error value for the target registration error which is also confirmed by the computation of the TRE values. This can be seen clearly in base region of the head. The TPS registered result has a darker shade of red in this region indicating a higher TRE compared to the corresponding region of the MLS registered result which has a lighter red shade.

While registering the 2D brain images, we used ABA [Rohde et al., 2003] for identifying the homologous control points. This opens the discussion as to whether systematic errors are present in the quantitative analysis. The adaptive bases algorithm uses compactly supported radial basis functions for registering two images. The TPS transformation also uses radial basis functions as its interpolating function. It is thus possible some systematic 
errors exist due to this use of radial basis functions but we have not performed any specific tests to confirm the presence or absence of any bias.

In conclusion, we found that the MLS transformation produced better results than the TPS technique both qualitatively and quantitatively. We also found that placing control points only in certain regions of the image does not cause the remaining regions to be non-uniformly deformed in the case of the MLS transformation while the location of control points is more important in case of the TPS transformation. The MLS transformation technique was found to work well for both CT and MR images. 


\section{CHAPTER V}

\section{EVALUATION OF THREE DIMENSIONAL REGISTRATION USING MLS AND TPS ALGORITHMS}

This chapter describes the method and results for performing $3 \mathrm{D}$ registrations using the MLS and TPS methods.

\section{V.1 Method}

We used two whole body CT volumes obtained from two different mice for the source and target volumes. The volumes were $512 \times 512 \times 512$ in size with $02 \times 0.2 \times 0.2$ $\mathrm{mm}^{3}$ voxel resolution. To reduce computation time we down-sampled these volumes to $128 \times 128 \times 128$ in size. CT volumes obtained from different mice provides a good dataset for non rigid registration due to the presence of anatomical difference in the structures of the mice. We performed the evaluation of the two methods first on five slices and then on ten slices obtained from the source and target volumes. To obtain the slices we selected five consecutive slices from the source volume, which showed maximum detail. We selected slices in which the head, spine and legs were most visible. This selection process was repeated for the target volumes to obtain the five slices. Control points for the registration process were selected manually on all of the five slices of the source volume. CT volumes show the bony structures in the body more prominently than soft tissues. Thus we selected control points on the bony structures present in head and spine regions of the five slices in the source volume. Similarly the homologous control points on the 
five slices in the target volume were also selected manually on the bony structures in the head and spine regions, thus giving a set of homologous control points for the registration to be performed. Figure 5.1 (a) and (b) show the four slices of the source and target volumes. Figure 5.1 (c) shows the result of registering the two volumes using the MLS technique and Figure 5.1 (d) shows the result of registration using the TPS technique. The results are discussed in detail in the section V.2.

For our next evaluation we selected ten slices from the source and target volumes showing maximum details. In this case we segmented the bony structures from the ten slices by setting an intensity threshold value. This made the control point selection easier and seemed a natural modality for control point selection. As in the previous case control points were picked manually on the source volume across the ten slices. The control points were placed in the bone structures of the head and spine. The corresponding homologous control points on the target volume were also picked manually giving us a set of control point for the source and target volumes. Registration was performed using the MLS and TPS techniques. Figure 5.2 (a) and (b) shows four slices from the source and target volumes respectively. Figure 5.2 (c) shows the result of the MLS registration method superimposed on to the four slices from the target volume and Figure 5.2 (d) shows the results of the TPS registration method superimposed on to the four slices of the target volume. The superimposed results give a better visual comparison of the two techniques. The results are discussed in detail below. 


\section{V.2 Results}

Figure 5.1 (c) and (d) show the result of registration using the MLS and TPS techniques. We found that the MLS algorithm performs better than the TPS method qualitatively. Comparing the slice one in Figure 5.1 (c) and (d), it was observed that the MLS transformation does not show any non-uniform stretching while the TPS transformation shows stretching near the head of the mouse as well as in the region near the end of the spine and tail. This stretching was observed in all the slices. Observing slice one of Figure 5.1(d) showed that head of the mouse to be more stretched than in the other slices. This unnatural stretching is not seen in the slices of the MLS registered volume. Due to the as-rigid-as-possible nature of transformation of the MLS method the overall rigidity is maintained while local deformation is also achieved. It was observed that the MLS technique maps the homologous points exactly and aligns the source volume as closely as possible to the target volume while retaining the rigidity at the same time. The TPS method though maps the homologous points exactly too but does not have control over the rigidity of the object and causes a lot of stretching.

Figure 5.2 (c) and Figure (d) show four slices from the result of the MLS and TPS algorithms along with four slices from the target volume. It was observed that the MLS method outperforms the TPS method in this case too. It was easier to compare the two methods when only the bony structures were used in the registration. The MLS registration result was found to be more in alignment with the target volume. This was observed especially in the spine area. Compare slices 2, 3 and 4 from Figure 5.2 (c) to Figure 5.2 (d). The TPS versions in 5.2 (d) are stretched in opposite directions unacceptably because this is a bony structure and should not deform. Looking at slices 
two, three and four in Figure 5.2 (c) and (d), the region near the end of the spine showed a stretching, that looks like the spine was stretched in opposite directions, in the TPS registered volume. The MLS transformation was observed to map the source volume to the target volume without this unnatural stretching. The head regions in both the results were found to align equally well. Control points were places only on the head and spine regions and hence the leg was not controlled in either method. Quantitative comparisons were not carried out as it was difficult to obtain the exact homologous points in the course and target volumes. Any error in the homologous points would result in an incorrect value of the target registration error.

Based on the results obtained we concluded that the MLS algorithm outperforms the TPS algorithm qualitatively. The MLS technique is able to preserve the overall rigidity of the object while still performing local non-rigid deformations. The TPS algorithm was found to cause unwanted stretching. This is unacceptable in cases where the rigidity of the object needs to be maintained especially areas such as bones which need to maintain their rigid structures. 


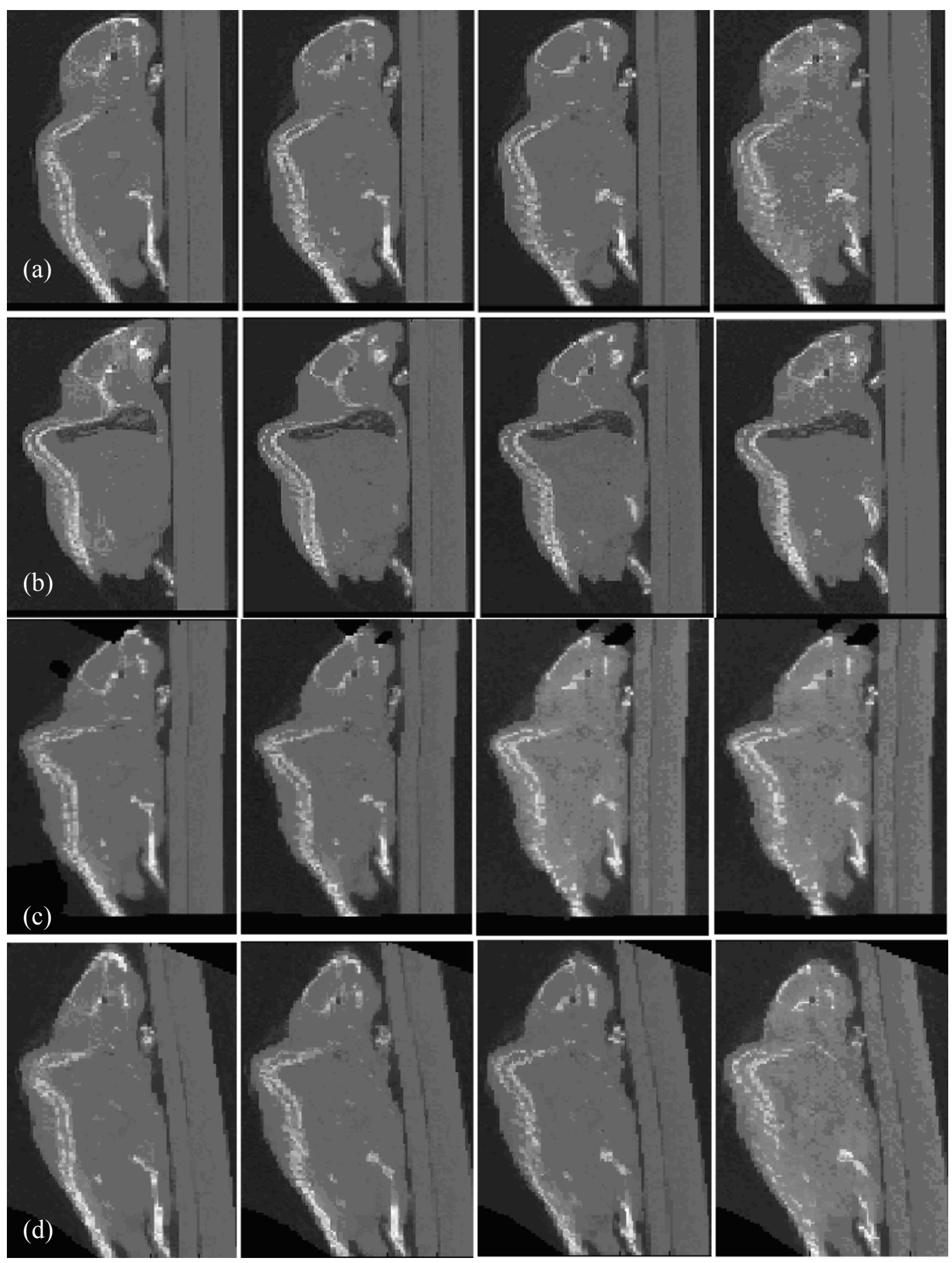

Figure 5.1 Registration of 3D CT volumes of mice. (a),(b): 4 slices from source and target volumes (c) 4 slices from MLS registered result volume. (d) 4 slices from TPS registered volume. The MLS technique performs better than the TPS technique by minimizing the non-uniform scaling and stretching. 

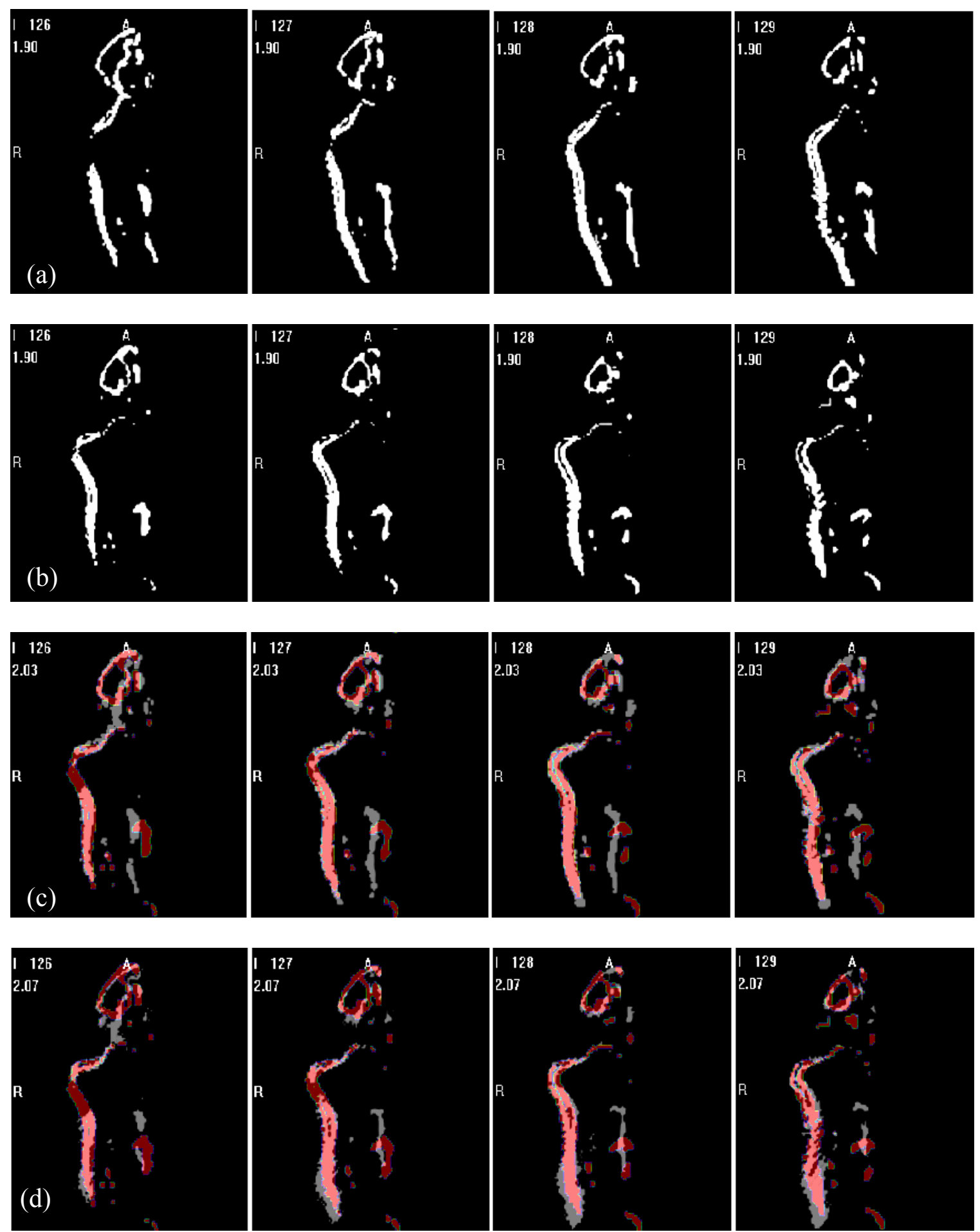

Figure 5.2 (a) 4 slices from source volume. (b) 4 slices from target volume. (c) 4 slices from MLS registered result superimposed on 4 slices of target volume. (d) 4 slices from TPS registered result superimposed on 4 slices of target volume. The MLS method clearly outperforms the TPS technique which shows a lot of stretching in the spine region. Control points were placed on the head and spine (thus the leg was not controlled for in either method. 


\section{CHAPTER VI}

\section{CONCLUSION AND FUTURE WORK}

We have compared two interpolating non-rigid registration techniques, Moving Least Squares (MLS) and Thin-Plate Splines (TPS). Both quantitatively and qualitatively we have found that the MLS technique outperforms the TPS technique for a given number of control points. A theoretical nicety of the MLS technique is that the deformation field is as rigid as possible, given the constraint that the control points are to be interpolated. This can offer advantages over other non-rigid registration methods, particularly those where bony structures should be minimally deformed during registration. Non-rigid registrations often produce unwanted stretching in the images and the unpredictable nature of the deformation field poses a major drawback which makes an algorithm with the ability to produce as-rigid-as-possible transformations attractive. The as-rigid-as-possible nature of the MLS technique thus makes it a suitable candidate for non-rigid registrations as it provides a transformation that maintains the rigidity of structures that need to remain non-deformed, while producing local deformations.

Chapters VI present the results of evaluation of the two non-rigid registration techniques for 2D CT images of whole body mice and MR images of human brain. The results show that the MLS algorithm performs qualitatively better than the TPS algorithm. The overall rigidity of the image is maintained better with the MLS method. This can be seen clearly in the example of the 2D CT mice images. The bone structures in the head and spine of the mouse in the source image is deformed to match the target image while 
the other regions of the image remain minimally affected thus maintaining the global rigidity while performing local deformations. In case of the TPS method, the results show unwanted stretching and shearing in the image. This unwanted stretching is caused due to the effect of the control points on the overall deformation field. Each point has a global effect on the transformation and thus affects the overall rigidity of the image.

The results for the registration of $2 \mathrm{D}$ MR human brain images show that both methods perform equally well. It is difficult to judge the better method visually due to the numerous structures in the brain. A quantitative evaluation by comparison of the target registration error shows that the MLS method has lower error values. The average of the target registration errors over 25 trials also show that the MLS algorithm performs better. The t-test results for the mean of the TRE values show that the mean is significantly different for the MLS method.

Chapter $\mathrm{V}$ presents and discusses the results for the 3D registration using the MLS and TPS methods. Again the results show that MLS algorithm outperforms the TPS algorithm qualitatively maintaining the rigidity as much as possible but still producing non-rigid deformations to map the source and target volumes.

Selecting the control points forms an important aspect of image registration. Thus it becomes necessary to select these control points as accurately as possible. Any error in this placing of points affects the final registration result. This is more pronounced in case of the TPS transformation as each point has a global effect on the deformation field. From the results obtained for the MLS transformation we observed that the as-rigid-aspossible nature of transformation produces an acceptable deformation even if one or two control points are not placed accurately. This is because of the weighting function on the 
least squares error function. This weighting function ensures that the effect of the control point in regions far away from it is less affected. The MLS transformation involves computing the transformation at each point in the image or volume. This leads to a longer computation time though it produces good results. One way to reduce the computation time would be to decimate the image/volume with a grid and computing the transformation only at the grid vertices and interpolating the other point in the image using an interpolation technique like bilinear interpolation.

The MLS method can be used for producing better registration results. In the future we would like to evaluate the performance of the MLS algorithm by modifying the weight parameter. The deformation field can be controlled by varying the value of alpha in the weight function. We would also like to examine the results by using different distance functions, other than the Euclidean distance used here, to see the variation of rigidity in the deformation. Another area of interest would be to study the effect of perturbation of the control points. It would be interesting to how much the control points can be perturbed while still producing a good transformation. Another way to optimize the MLS algorithm would be to automate the process of control point selection. This work would be on the line of Chui [Chui et al. 2003]. Automating the process would result in a better alignment of the registered result and target volume in case of 3D applications. Similarly including an iterative approach would also result in optimizing the moving least squares algorithm. 


\section{REFERENCES}

[1]. M. Alexa, D. Cohen-OR, D. Levin, "As-Rigid-As-Possible Shape Interpolation", In Proceedings of ACM SIGGRAPH, pp. 157-164, 2000.

[2]. F. Bookstein, "Principal Warps: Thin-Plate Splines and the Decomposition of Deformations", IEEE Transactions on Pattern Analysis and Machine Intelligence, vol. 11, no. 6, pp. 567-585, Jun. 1989.

[3] H.Chui and A. Rangarajan, "A New Point-Matching Algorithm for Non-Rigid Registration", Computer Vision and Image Understanding, pp. 114 -141, 2003.

[4]. W.R. Crum, T. Hartkens, and D.L.G. Hill, "Non-rigid Image Registration: Theory and Practice”, The British Journal of Radiology, vol. 77, pp. S140 - S153, Dec. 2004.

[5]. A. Cuno, C. Esperança, A. Oliveira, and R. Cavalcanti, "3D As-Rigid-As-Possible Deformations Using MLS”, Proc. Computer Graphics International Conference, May 2007.

[6]. P.A. Freeborough and N.C. Fox, "Modeling brain deformations in Alzheimer disease by fluid registration of serial 3D MR images", Journal of Computer Assisted Tomography, vol. 22, pp. 838-843, 1998.

[7]. A. Goshtasby, "Registration of Images with Geodesic Distortion", IEEE Transactions on Geoscience and Remote Sensing, vol. 26, no. 1, pp. 60-64, Jan. 1988.

[8]. A. Goshtasby , "2D and 3D Medical Registration for Medical, Remote Sensing, and Industrial Applications", April 2005.

[9]. J. Hajnal, D. Hill, and D. Hawkes, "Medical Image Registration”, CRC Press, 2001, pp 120-121.

[10] T. Igarashi, T. Moscovich, and J.F. Hughes, "As-Rigid-As-Possible Shape Manipulation", ACM Transactions on Graphics, vol. 24, no. 3 pp. 1134-1141, 2005.

[11]. H. J. Johnson and G. E. Christensen, “Landmark and Intensity Based, Consistent Thin-Plate Spline Image Registration", Proc. of International Conference on Information Processing in Medical Imaging, vol. 2082, pp. 329-343, 2001.

[12] R. Kolluri, "Provably good moving least squares", Proc. ACM-SIAM Symposium on Discrete Algorithms, pp. 1008 - 1017, 2005.

[13] J.B. Maintz and M.A. Viergever, "A Survey of Medical Image Registration", Medical Image Analysis, vol. 1, no. 1, pp. 1-36, Mar. 1998. 
[14]. H. Park, P.H. Bland, and C.R. Meyer, "Construction of an abdominal Probabilistic atlas and its application in segmentation", IEEE Transactions on Medical Imaging, vol. 22, no. 4, pp 483-492, Apr. 2003.

[15]. G.K. Rohde, A. Aldroubi, and B.M. Dawant, "The Adaptive Bases Algorithm for Intensity-Based Non-Rigid Image Registration”, IEEE Transactions on Medical Imaging, vol. 22, no. 11, pp.1470- 1479, Nov. 2003.

[16]. K. Rohr, H. S. Stiehl, R. Sprengel, T. M. Buzug, J. Weese, and M. H. Kuhn: "Landmark-Based Elastic Registration Using Approximating Thin-Plate Splines". IEEE Transactions on Medical Imaging, vol. 20, no. 6, pp. 526-534, 2001.

[17]. K. Rohr, M. Fornefett, H. S. Stiehl, "Spline-based elastic image registration: integration of landmark errors and orientation attributes", Computer Vision and Image Understanding, vol. 90 no. 2, pp. 153-168, 2003.

[18]. F. Sauer, “ Image Registration: Enabling Technology for Image Guided Surgery and Therapy", Engineering in Medicine and Biology Society, pp. 7242-7245, 2005.

[19]. S. Schaefer, T. Mcphail, and J. Warren, "Image deformation Using moving least squares”, ACM Transactions on Graphics, vol. 25, no. 3, pp. 533-540, Jul. 2006.

[20]. M. Sonka and M. Fitzpatrick, "Handbook of Medical Imaging”, vol. 2, Medical Image Processing and Analysis, SPIE Press Monograph, Chapter 8, pp. 465-470, 2000.

[21]. A.W. Toga and P.M. Thompson, "Image registration and the construction of multidimensional brain atlases", Handbook of medical imaging, pp. 635-653, 2000.

[22]. Y. Zhu and S.J. Gortler, "3D Deformation Using Moving Least Squares", Harvard Computer Science Technical Report: TR-10-07, 2007. 